\title{
Mejora del proceso de forja mediante la aplicación de nitruración a alta presión y la introducción de una lubricación ecológica
}

\author{
J. BARRIGA ${ }^{1}$; A. IGARTUA ${ }^{1}$; M. MARÍN ${ }^{1}$ J.L. ROMERO ${ }^{2}$, I. GORDOA ${ }^{3}$; A. MONCLÚS ${ }^{4}$, D. PLANELL ${ }^{4}$ \\ ${ }^{1}$ Fundación TEKNIKER, 20600 Eibar, Gipuzkoa \\ ${ }^{2}$ GSB Forja, 20230 Legazpi, Gipuzkoa \\ ${ }^{3}$ FPL-GKN, 20230 Legazpi, Gipuzkoa \\ ${ }^{4}$ FUCHS Lubricantes, 01013 Vitoria
}

\begin{abstract}
Una de las técnicas más empleadas para mejorar la vida de las herramientas de conformado es la nitruración. En el presente estudio se ha empleado la tecnología de nitruración gaseosa a alta presión aplicada a utillaje de forja en semicaliente y calibrado en frío. La utilización de presiones elevadas potencia el efecto nitrurante de la atmósfera de $\mathrm{NH}_{3}$, por lo tanto presenta una serie de ventajas frente a otras técnicas convencionales. Además, en el marco de este trabajo, se han desarrollado diferentes lubricantes ecológicos para su utilización en estas aplicaciones de conformado y se ha analizado la interacción de estos productos con las superficies nitruradas. En concreto se ha estudiado la introducción de un lubricante grafitado en base vegetal para forja en semicaliente y de varios aceites fosfolubricantes para piezas que sufrirán una transformación en frío. Este análisis se ha llevado a cabo inicialmente en el laboratorio mediante diversos ensayos tribológicos y finalmente en pruebas de campo.
\end{abstract}

Palabras clave: forja, nitruración, lubricación ecológica, tribología.

Improvement of forging processes using high pressure nitriding and ecological lubrication.

Nitriding is one of the most used techniques to enhance the life in service of forming tools. In this study high pressure gas nitriding technology has been applied in warm and cold forming tools. The utilisation of high pressure enhances the nitriding effect of the $\mathrm{NH}_{3}$ atmosphere and several advantages arise comparing with conventional techniques. Besides of this, in the framework of this project, several ecological lubricants have been developed to be used in both forming applications and the interaction of these products with the nitrurated surfaces has been study. Specifically, it was studied the introduction of a vegetable oil with graphite for warm forging and several phosphorlubricating oils to be applied in pieces for a cold forming transformation. In a first stage this analysis was carried out in a laboratory by means of tribological tests and lately in field tests.

Keywords: Forging, nitriding, ecologic lubrication, tribology.

\section{INTRODUCCIÓN}

El presente artículo es el resultado del trabajo desarrollado en un proyecto de investigación centrado en un proceso de forja básicamente con un objetivo ecológico: reducir el impacto medioambiental de los lubricantes y tratamientos superficiales empleados.

GSB-Forja y Forjas de Precisión de Legazpia (GKN) son dos fabricantes de productos forjados para la industria de Automoción. En ambas empresas se fabrican tulipas y manguetas combinando un proceso de forja en semicaliente (el material entra a unos $850^{\circ} \mathrm{C}$ ) y un calibrado en frío (operación final de conformado a temperatura ambiente) con objeto de conseguir las tolerancias requeridas en el producto.

Principalmente se han desarrollado tres líneas de trabajo diferentes:

-Sustituir el lubricante grafitado en base mineral que se utiliza para el proceso en semicaliente por otro en base vegetal, biodegradable y no tóxico.

-Reemplazar el tratamiento lubricante de fosfatado de zinc y jabonado del calibrado en frío por un aceite derivado del ácido fosfórico.

-Incorporar el tratamiento de nitruración gaseosa a alta presión (NAP) en las herramientas para incrementar su vida útil y sustituir los baños en sales cianuradas.

Evidentemente, además de exigir que los nuevos lubricantes fabricados en Fuchs sean medioambientalmente inocuos, se requieren buenas cualidades técnicas. El método ideal para llevar a cabo dicha evaluación es mediante pruebas de campo, pero son excesivamente costosas. Es por ello que previamente se ha realizado una preselección mediante ensayos tribológicos (caracterización de fricción y desgaste) en los laboratorios de TEKNIKER.

\section{PARTE EXPERIMENTAL}

\subsection{Nitruración gaseosa a alta presión}

Entre los factores que más afectan a la vida útil de las herramientas de conformado se encuentra el desgaste superficial producido por la fricción y la fatiga mecánica. Una de las técnicas más empleadas para mejorar la vida de las herramientas de conformado es la nitruración. La nitruración es un proceso termoquímico de enriquecimiento superficial de nitrógeno realizado en fase ferrítica sobre los aceros que aumenta la resistencia al desgaste y la vida a fatiga de herramientas y elementos de máquinas.

Los procesos de nitruración se componen de dos mecanismos:

1. Un tratamiento de conversión química superficial que conduce a la formación, en la superficie de la pieza, de una capa de combinación (capa blanca o capa de compuesto) compuesta de nitruros de hierro. Esta capa blanca puede tener varias estructuras:

- Capa monofase $\gamma^{\prime}\left(\mathrm{Fe}_{4} \mathrm{~N}\right)$ de estructura cúbica centrada en la cara. 
- Capa monofase $\varepsilon\left(\mathrm{Fe}_{3-2} \mathrm{~N}\right)$ de estructura hexagonal compacta.

- Capa bifase formada por una combinación $\gamma$ y $\varepsilon$.

2. Un tratamiento de difusión del nitrógeno en solución sólida intersticial y de combinación con ciertos elementos de aleación procedentes del acero que se va a tratar, en nitruros finamente dispersos conduciendo a un endurecimiento por reforzamiento estructural en una profundidad llamada capa o zona de difusión.

Existen diferentes técnicas de nitruración, pero fundamentalmente se emplean tres: nitruración gaseosa (convencional y alta presión), iónica y por sales. La nitruración a alta presión es una variante de la nitruración gaseosa convencional pero en la que se trabaja a presiones superiores. Aumentando la presión se reduce el grado de disociación del amoniaco en la atmósfera nitrurante y por lo tanto es mayor la probabilidad de que esta disociación se produzca sobre las piezas (solo el nitrógeno naciente que se produce sobre las piezas es el que nitrura), resultando en un aumento del potencial nitrurante. Los méritos de los procesos de nitruración a alta presión son particularmente visibles cuando las piezas poseen formas extremadamente complejas con agujeros y cavidades pequeñas que deben ser nitruradas. Otras ventajas que posee la tecnología de nitruración a alta presión son las siguientes:

- Medioambientalmente aceptable.

- No requiere limpieza posterior de las piezas tratadas.

- Buen aprovechamiento del espacio útil de la cámara.

- Reducción del tiempo de tratamiento.

- Bajo consumo de gas.

- Fácil nitruración de aceros altamente aleados y aleaciones de titanio

- Control de la estructura de la capa blanca.

- Minimización de la limpieza previa de las piezas.

Los materiales que se han nitrurado han sido aceros que se emplean en herramientas para trabajo en semicaliente (H10 y H13) y para forja en frío (M2). Se han empleado varios procesos ligeramente diferentes, pero con unas características comunes: temperaturas de $500-600^{\circ} \mathrm{C}, \mathrm{NH}_{3}(6$ bar $)$ y $\mathrm{N}_{2}(2$ bar $)$.

Tras un estudio metalográfico de las piezas nitruradas en los discos se han obtenido los resultados que se recogen en la Tabla 1 y la Figura 1.

TABLA 1: ESPESOR DE LA ZONA DE DIFUSIÓN

\begin{tabular}{|c|c|}
\hline \multicolumn{2}{|c|}{ Espesor de zona de difusión (mm) } \\
\hline Disco M2 & 0,24 \\
\hline Disco H 10 & 0,37 \\
\hline
\end{tabular}

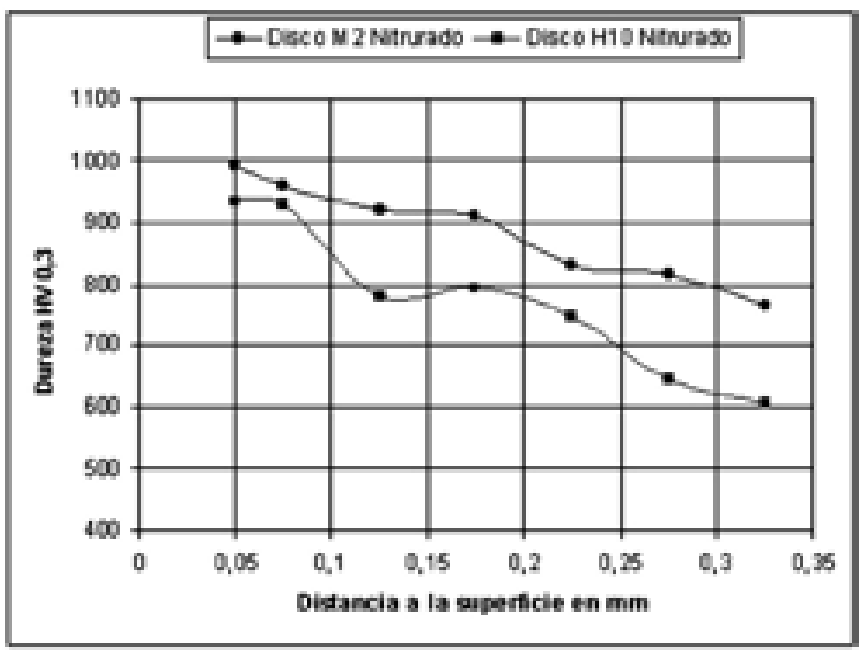

Figura 1: Cadena de microdurezas
En las Fotografías 1 y 2 se pueden ver secciones transversales de las piezas en las cuales se aprecia que la zona de difusión es mayor en el material H10 que en el M2.

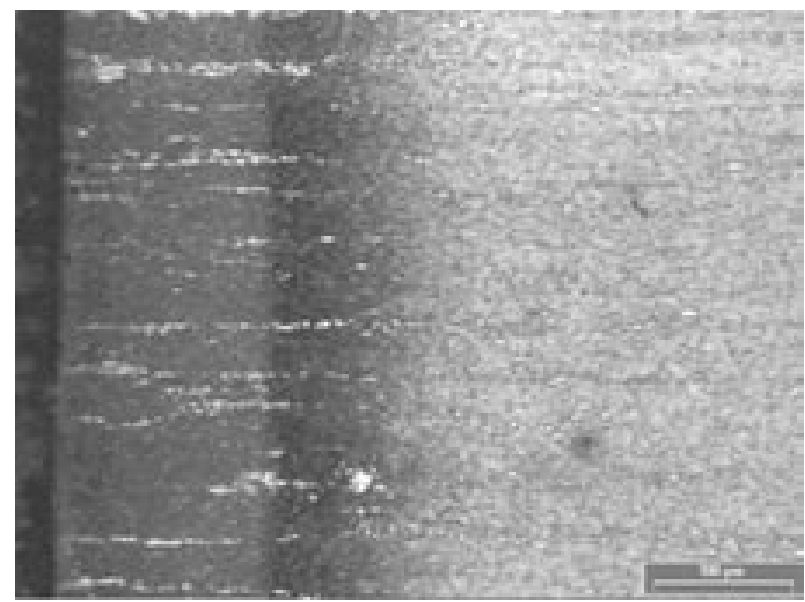

Fotografía 1: Sección M2

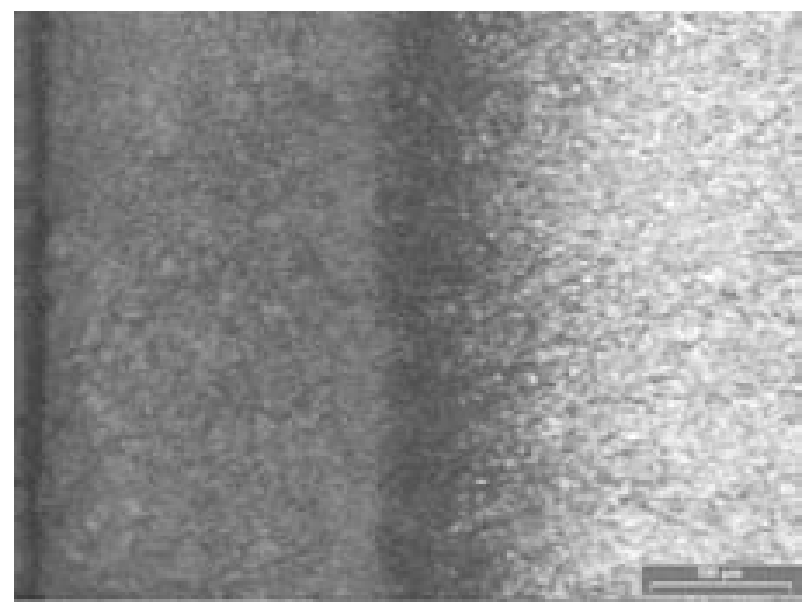

Fotografía 2: Sección H10

Para el tratamiento de los punzones y matrices de FPL y GSB se ha realizado procesos de nitruración ampliados con 6 cargas de amoníaco. Esto se ha realizado así debido a que cuando se estudiaron metalográficamente punzones probados en planta se observó que la zona afectada térmicamente era importante. Por lo tanto, se persiguió aumentar la profundidad de la capa de nitruración. Como consecuencia de este tratamiento el espesor de capa de nitruración obtenida ha alcanzado una profundidad de aproximadamente $0.45 \mathrm{~mm}$.

\subsection{Lubricantes ecológicos}

La empresa FUCHS se ha encargado de desarrollar lubricantes ecológicos para forjar en semicaliente (lubricantes A) y en frío (lubricantes B). Los objetivos medioambientales que nos fijamos para todos los productos desarrollados en el marco de este proyecto, tanto para la aplicación de forja en semicaliente como para la de calibrado en frío, son los siguientes:

- No puede contener elementos cancerígenos ni mutagénicos.

- No puede contener ni cloro en enlaces orgánicos ni nitruros.

- No puede contener metales.

- Biodegradabilidad $>80 \%$ según el ensayo CEC-L-33-A-93.

- Toxicidad >100mg/1 según el ensayo OECD 202 Daphnia Magna. 
En las tablas 2 y 3 se pueden ver los resultados de la caracterización medioambiental de los lubricantes. Se aprecia que el lubricante grafitado A2 no cumple con los requisitos de biodegradabilidad y el aceite B2 es ligeramente tóxico.

TABla 2: BiodegradabiLidad. Test CEC L33-A-95

\begin{tabular}{|c|c|}
\hline Lubricante & \%BIO \\
\hline Fuchs A1 & 81 \\
\hline Fuchs A2 & 76.7 \\
\hline Fuchs A3 & 98 \\
\hline
\end{tabular}

TABla 3: OECD 202, Daphnia Magna, TOXICITY TeST

\begin{tabular}{|c|c|c|}
\hline Lubricante & EL50(mg/l) & Clasificación \\
\hline Fuchs A1 & $>1000$ & Relativamente inocuo \\
\hline Fuchs A2 & $>1000$ & Relativamente inocuo \\
\hline Fuchs A3 & $>1000$ & Relativamente inocuo \\
\hline Fuchs B1 & 222 & Prácticamente no tóxico \\
\hline Fuchs B2 & 99 & Ligeramente tóxico \\
\hline
\end{tabular}

Uno de los mayores problemas que pueden surgir con los lubricantes vegetales es su baja resistencia a la temperatura. Para asegurar que esta situación estuviera controlada con los nuevos lubricantes, hemos realizado un análisis mediante DSC (calorimetría diferencial de barrido) en el que se analiza la temperatura a la cual comienza la degradación del lubricante (tabla 4). Hemos comparado los valores obtenidos con los lubricantes vegetales con los resultados de varios lubricantes minerales grafitados (M1, M2 y MR -lubricante reciclado-). Se aprecia que, efectivamente, los lubricantes en base mineral se degradan a temperaturas mayores que los de base vegetal. Sin embargo, con uno de los lubricantes ecológicos (A3) se obtiene un valor similar al del lubricante mineral reciclado (MR), que es un lubricante que sabemos que funciona correctamente.

TABLA 4: ENSAYO DINÁMICO EN DSC: TEMPERATURA DE COMIENZO DE LA DEGRADACIÓN $\left({ }^{\circ} \mathrm{C}\right)$

\begin{tabular}{|l|c|c|c|c|c|c|}
\hline Lubricante: & M1 & M2 & MR & A1 & A2 & A3 \\
\hline $\mathbf{T}^{\mathbf{a}}{ }^{\mathbf{}} \mathbf{C}$ ): & 238 & 197 & 172 & 146 & 150 & 177 \\
\hline
\end{tabular}

Los aceites desarrollados para el conformado en frío, son productos que reaccionan por inmersión de las piezas que se van a procesar. Durante la inmersión, dichos lubricantes reaccionan con el acero produciendo en la superficie una capa de fosfatos de hierro que actuará de lubricante durante el proceso de conformado. La eficacia de dicho tratamiento depende de la calidad superficial de las piezas tratadas y varía con la temperatura y el tiempo de inmersión.

\subsection{Ensayos Tribológicos}

En los ensayos de laboratorio llevados a cabo en TEKNIKER se han examinado las características técnicas de los lubricantes y del tratamiento de nitruración desde el punto de vista de la fricción y el desgaste. Los lubricantes ecológicos desarrollados para sustituir el tratamiento de fosfatado de cinc en el calibrado en frío (Fuchs B) han sido comparados también con varios aceites fosfolubricantes (no ecológicos) existentes en el mercado (oil 1,2,3). La inmersión en estos productos ha sido de $10 \mathrm{~min}$ a $30^{\circ} \mathrm{C}$ y a $60^{\circ} \mathrm{C}$.

En los ensayos Pin on Disc (POD) se han utilizado pines de acero F114. Para los ensayos de lubricantes grafitados los discos se han fabricado en $\mathrm{H} 10$ y para simular el calibrado en frío se han utilizado discos de M2.

Se observa que con los pines nitrurados, en general, el desgaste y el coeficiente de fricción disminuyen (figs. 2 y 3), excepto en el caso del coeficiente de fricción con el lubricante M2 y el desgaste con el M1. También se aprecia una tendencia de menor rozamiento usando los lubricantes en base vegetal. El menor desgaste se ha producido usando el material nitrurado y el lubricante en base vegetal A3.

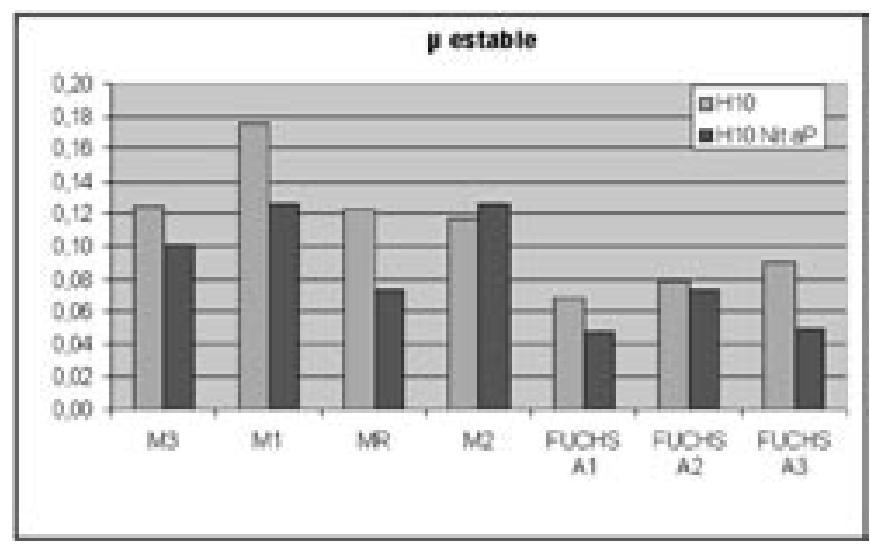

Figura 2: Coeficiente de fricción estable con configuración Pin on Disc para forja en semicaliente

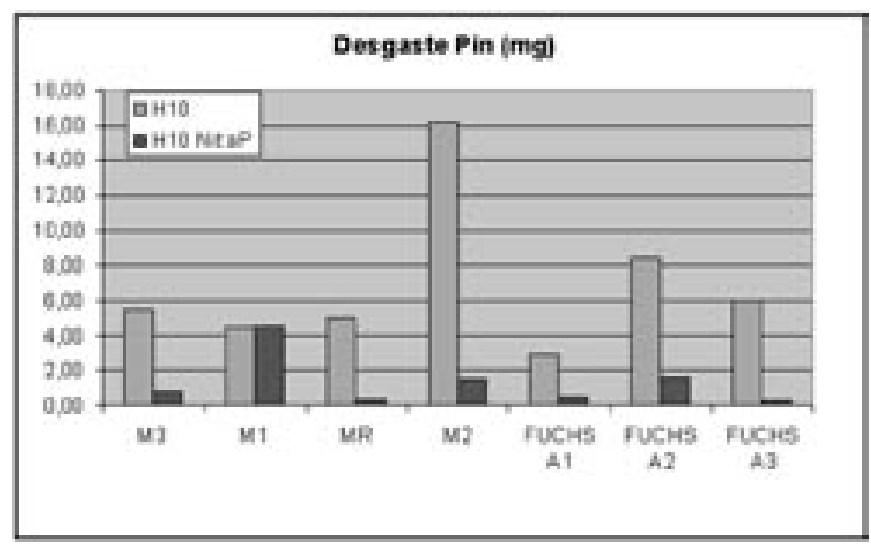

Figura 3: Desgaste del pin para forja en semicaliente

En los ensayos POD para simular el calibrado en frío se han realizado todos los ensayos con discos de M2 nitrurados salvo en la referencia (figs. 4 y 5). El desgaste con el lubricante Fuchs B2 $\left(30^{\circ} \mathrm{C}\right)$ es extremadamente alto $(6.1 \mathrm{mg})$ y no aparece representado en la gráfica. Con el lubricante Fuchs B1 se obtienen menores coeficientes de fricción y desgastes que con el tratamiento de fosfatado de cinc y jabonado.

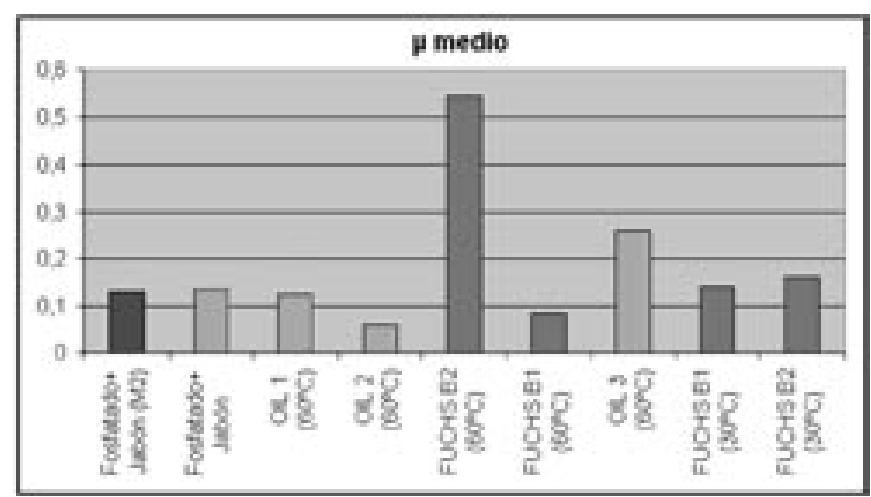

Figura 4: Coeficiente de fricción estable con configuración Pin on Disc calibrado en frío 


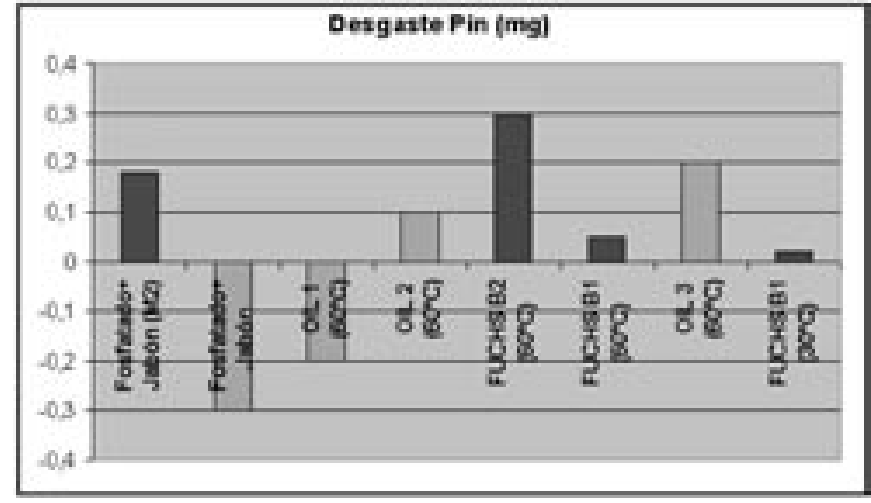

Figura 5: Desgaste del pin calibrado en frío

Los ensayos Rotational Drawing (RD) se han llevado a cabo con una máquina Falex Tapping Torque. Este equipo mide el par mientras un punzón girando conforma una chapa. Posteriormente, de esos valores de par se calcula la eficiencia de un fluido con respecto a la referencia. Las chapas utilizadas han sido de material F115. Para estudiar los lubricantes grafitados se han usado punzones de H13 usando como referencia el lubricante mineral M1 (eficiencia 100\%). Se aprecia (figs 6 y 7) que usando herramientas nitruradas prácticamente no hay diferencias entre lubricantes. Con H13 sin nitrurar, los lubricantes vegetales desarrollados obtienen una eficiencia superior a los lubricantes en base mineral.

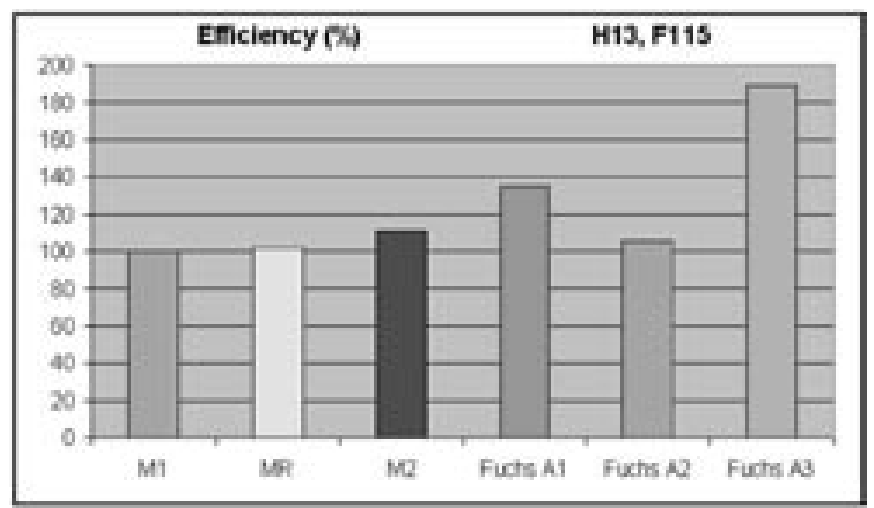

Figura 6: Eficiencia en ensayo Rotational Drawing para H13 sobre F115 en forja semicaliente

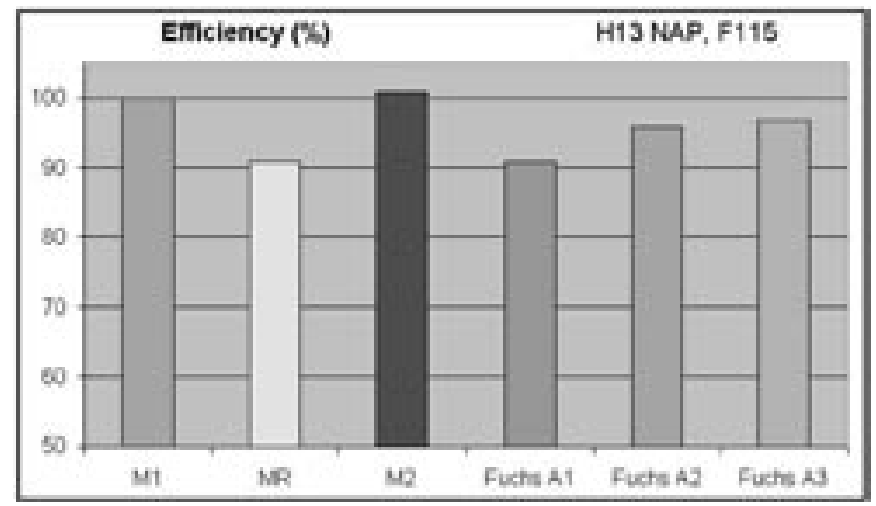

Figura 7: Eficiencia en ensayo Rotational Drawing para H13 NAP sobre F115 en forja semicaliente

Para comparar los tratamientos fosfolubricantes la referencia ha sido el fosfatado de cinc con jabonado. Utilizando punzones de M2 se aprecia que todos los lubricantes tienen una eficiencia mayor que la referencia cuando se realiza un tratamiento a $60^{\circ} \mathrm{C}$ (fig. 8). En el caso del M2 NAP, se obtiene también la mayor eficiencia con los lubricantes Fuchs B a $60^{\circ} \mathrm{C}$ (fig. 9). Se aprecia que la eficiencia disminuye con la temperatura de inmersión. Con el lubricante B1 la menor eficiencia (mayor par) se ha obtenido cuando se ha aplicado de forma directa sobre la pieza (sin sumergirla previamente).

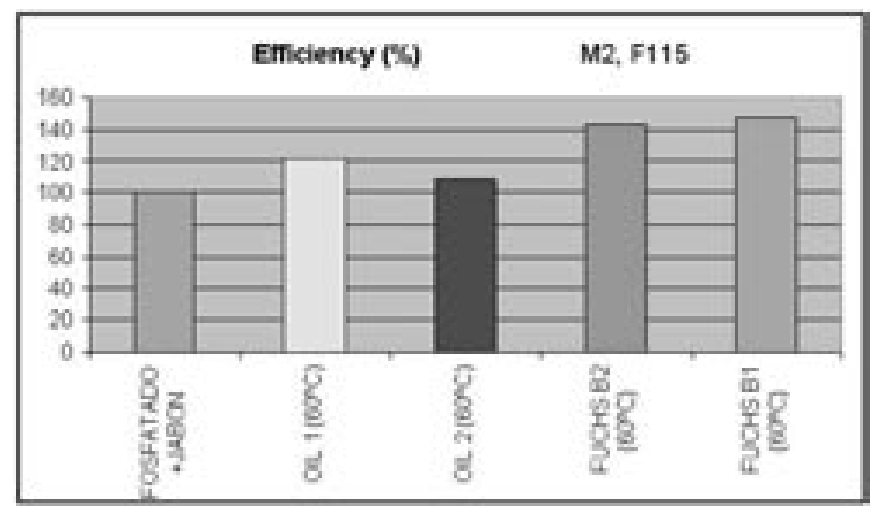

Figura 8: Eficiencia en ensayo Rotational Drawing para M2 sobre F115 calibrado en frío

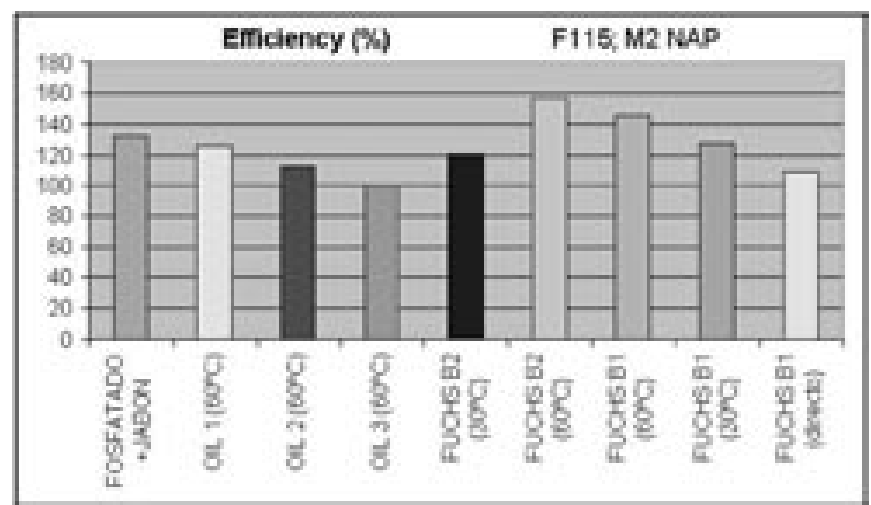

Figura 9: Eficiencia en ensayo Rotational Drawing para M2 NAP sobre F115 calibrado en frío

Para comparar las características de extrema presión de los lubricantes se ha realizado el ensayo de Cuatro Bolas ASTM 2783. En los resultados (fig. 10) se aprecia que tanto los lubricantes vegetales como el aceite mineral reciclado (MR) soportan cargas superiores que los lubricantes grafitados en base mineral. Esta diferencia probablemente es debida a la aditivación empleada.

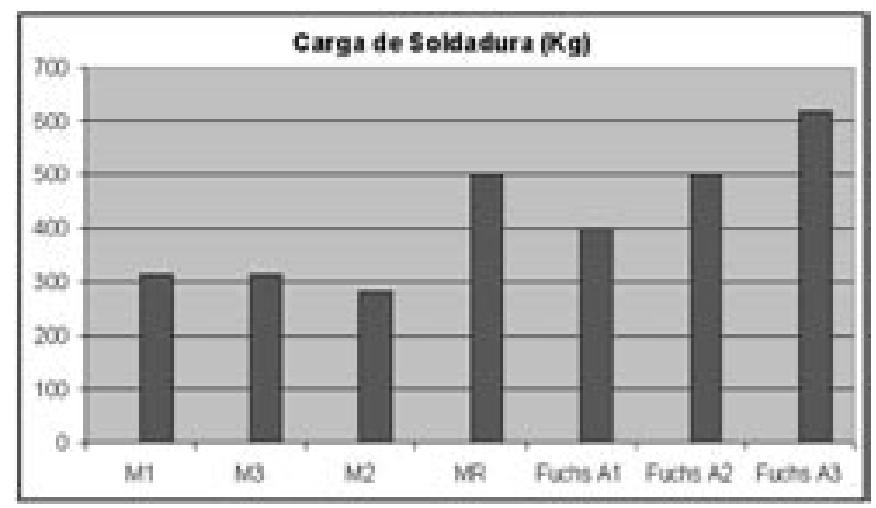

Figura 10: Ensayo de Cuatro Bolas 


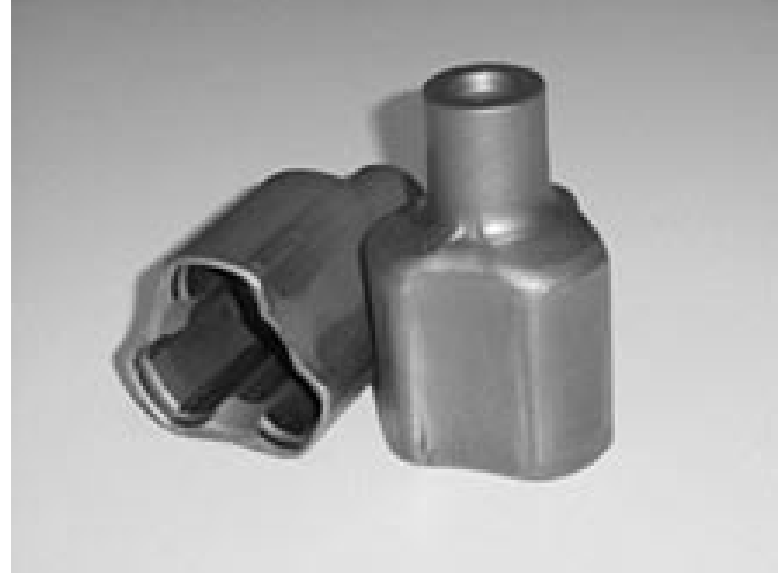

Fotografía 3: Tulipas. Producto acabado

\subsection{Pruebas industriales}

Como resultado de la fase de ensayos tribológicos y análisis de los nuevos lubricantes ecológicos desarrollados, se decidió llevar a cabo las pruebas en forja real con el lubricante vegetal grafitado Fuchs A3 en el proceso de forja en semicaliente y con el tratamiento fosfolubricante (aceite) Fuchs B1 como sustituto del fostatado de cinc y jabonado.

En FPL se han probado matrices y punzones de extrusión directa e inversa utilizando nitruración a alta presión en forja en semicaliente. Se consiguió, en una matriz de extrusión directa de la primera operación de una mangueta, una vida de 34567 piezas, siendo la vida media de este utillaje sin nitrurar de 19500-22000 piezas. En general, se ha obtenido una mejora de un $30 \%$ en la vida de matrices con respecto a la nitruración en sales. En los punzones no se han obtenido mejoras: las tensiones máximas se dan por debajo de la capa nitrurada y el incremento de dureza superficial no contribuye a un menor deterioro del utillaje.

En GSB-Forja se ha probado el lubricante Fuchs A3: grafitado en base vegetal para forja en semicaliente. Los resultados obtenidos han sido buenos, la producción ha sido la normal, es decir, la vida de utillaje no ha sufrido cambios. El único problema que surgió fue que este lubricante es más espeso que el mineral, lo cual hace más complicada su manipulación. Este problema ha sido finalmente solventado mediante una reformulación del lubricante.

Para el calibrado en frío se han realizado pruebas en GSB-Forja con el lubricante Fuchs B1 manteniendo las piezas en inmersión diferentes tiempos a temperatura ambiente y con distintos estados superficiales:

1. 24 horas de inmersión de piezas sin granallar

2. 24 horas de inmersión de piezas granalladas

3. 12 horas de inmersión de piezas granalladas

La prueba 2 fue un éxito, ya que tanto el conformado como la expulsión fueron correctos, detectando un aumento de la presión de trabajo no superior al 15\%. El aspecto final de las piezas también ha mejorado (Fotografía 3) y no es necesario realizar una limpieza posterior. Sería deseable reducir el tiempo de inmersión, para lo cual parece necesario activar la reacción elevando la temperatura.

\section{CONCLUSIONES}

La nitruración a alta presión es una alternativa ecológica a la nitruración en sales cianuradas. Se han probado matrices obteniendo mejoras de vida de hasta un $77 \%$ con respecto al utillaje sin nitrurar y un $30 \%$ con respecto a la nitruración por sales.

El proceso de nitruración a alta presión en punzones no mejora sustancialmente su vida útil.

La nueva aditivación A3 ha permitido aumentar considerablemente la resistencia a la temperatura de los lubricantes grafitados en base vegetal para forjar en semicaliente. Con el lubricante ecológico Fuchs A3 se han conseguido forjar piezas en prensas con una producción normal. Los aceites fosfolubricantes son una buena alternativa al fosfatado de cinc. Su reactividad aumenta con el tiempo de inmersión, con la temperatura y al granallar las piezas previamente. La mejora medioambiental es considerable ya que se eliminan baños de cinc contaminantes.

\section{AGRADECIMIENTOS}

Agradecemos al Gobierno Vasco y al Ministerio Español de Ciencia y Tecnología la financiación de este proyecto.

\section{BIBLIOGRAFÍA}

1. P. F. Bariani, "Wear in hot and warm forging: design and validation of a new laboratory test", Annals of the CIRP Vol 45/1/1996

2. Jung, M; Hoffmann, F; Mayr, P; Minarski, "High pressure nitriding" 2nd Int. Conf. on Carburizing and Nitriding, Ohio, 1995 p. 263-268

3. S. Komatsuzaki, T. Narahara, "Cold forming of steel with lubricating oils", Lubrication Engineering., 52, 259-266, 1996

Recibido: 1.2 .03

Aceptado: 30.11 .03 